\title{
Ensamble de crustáceos bentónicos en un lago salino tropical
}

\section{Benthic crustaceans assemblage in a tropical, saline lake}

\author{
Ma. del Carmen Hernández ${ }^{1}$, Elva Escobar ${ }^{2}$ y Javier Alcocer ${ }^{3 *}$

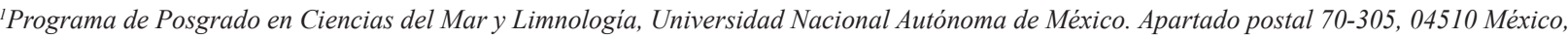 \\ D.F., México. \\ ${ }^{2}$ Laboratorio de Biodiversidad y Macroecología, Instituto de Ciencias del Mar y Limnología, Universidad Nacional Autónoma de México. Apartado \\ postal 70-305, 04510 México, D.F., México. \\ ${ }^{3}$ Proyecto de Investigación en Limnología Tropical, FES Iztacala, Universidad Nacional Autónoma de México. Av. de los Barrios 1, Los Reyes \\ Iztacala, 54090 Tlalnepantla, Estado de México, México. \\ *Correspondencia: jalcocer@servidor.unam.mx
}

\begin{abstract}
Resumen. El presente trabajo reconoce la composición, estructura y distribución espacial del ensamble de crustáceos bentónicos de Alchichica, un lago salino tropical ubicado en el extremo oriental del altiplano mexicano. El lago presenta una riqueza taxonómica de crustáceos bentónicos compuesta por 1 anfípodo (Hyalella azteca), 1 isópodo (Caecidotea williamsi) y 2 ostrácodos (Limnocythere inopinata y Candona sp.). Comparada con otros lagos tropicales, la riqueza de especies es reducida. A pesar de lo anterior, es importante mencionar el grado elevado de endemismo representado por $C$. williamsi, recientemente descrita para el lago Alchichica; adicionalmente, es factible que tanto Candona como $\mathrm{H}$. azteca sean especies nuevas y endémicas del lago. Los crustáceos bentónicos se distribuyen desde la zona litoral hasta la zona más profunda del lago $(62 \mathrm{~m})$ con abundancias y riqueza taxonómica variables. Los ostrácodos fueron los crustáceos que con mayor frecuencia se recolectaron en el lago, en la zona litoral, en el talud, y en la zona profunda de la que son habitantes exclusivos. Los anfípodos constituyeron el segundo grupo en abundancia de la zona litoral y talud y estuvieron ausentes en la zona profunda. Los isópodos sólo se encuentran asociados a los depósitos de tufa, hábitat característico del lago que se extiende a lo largo del talud, por lo que con las técnicas de muestreo tradicional empleadas en el presente estudio no fueron capturados. En este ensamble de crustáceos predominan las especies de desarrollo directo y con posiciones tróficas que incluyen componentes herbívoros (H. azteca), omnívoros (C. williamsi) y bacterívoros (L. inopinata y Candona sp.).
\end{abstract}

Palabras clave: Alchichica, México, ostrácodos, anfípodos, distribución, abundancia, biomasa.

\begin{abstract}
This work acknowledges the composition, structure and spatial distribution of the benthic crustaceans assemblage of Alchichica, a tropical saline lake located in the easternmost portion of the Mexican highlands. The benthic crustaceans' assemblage was comprised by 1 amphipod (Hyalella azteca), 1 isopod (Caecidotea williamsi) and 2 ostracods (Limnocythere inopinata and Candona sp.). Compared to other tropical lakes the species richness is low. It is worth mentioning the high endemism found in the benthic crustaceans' assemblage of Lake Alchichica exemplified by $C$. williamsi, a new and recently described endemic species; additionally, it is to be expected that both, Candona as $\mathrm{H}$. azteca are also new and endemic species of the lake. The benthic crustaceans were distributed from the coastal zone to the deepest part of the lake $(62 \mathrm{~m})$ displaying variable abundances and taxonomic richness. The ostracodes were the most common crustaceans collected in the littoral zone, in the slope and in the deep zone where no other species were found. Amphipods were the second most abundant group in the coastal zone and in the slope being absent from the deep zone. Isopods are associated exclusively with the tufa deposits, a characteristic habitat of the lake that runs along the slope, so that traditional sampling techniques like those used in this study were unable to collect them. The crustacean assemblage is predominantly composed by species with direct development of diverse trophic positions including herbivores $(H$. azteca), omnivores $(C$. williamsi) and bacterivores (L. inopinata and Candona sp.).
\end{abstract}

Keywords: Alchichica, Mexico, ostracods, amphipods, distribution, abundance, biomass.

\section{Introducción}

La presencia de lagos salinos en México es numerosa; no obstante, a pesar de su abundancia, distribución e

Recibido: 16 junio 2010; aceptado: 21 junio 2010 importancia (Alcocer y Williams, 1993), no han atraído la atención de los limnólogos, salvo algunas excepciones notables como Texcoco y Cuitzeo, y tan sólo uno, Alchichica, forma parte de los sitios de la Red Mexicana de Estudios Ecológicos a Largo Plazo (www.mexlter.org. $\mathrm{mx}$ ) con más de 10 años de monitoreo continuo. 
De acuerdo con el balance de Shiklomanov (1990), a nivel mundial la cantidad de aguas salinas epicontinentales es tan sólo ligeramente inferior a la de las dulces $(0.006 \%$ y $0.007 \%$, respectivamente). Aunque no se cuenta con la información precisa, es muy probable que en México esas proporciones se mantengan. Hay 2 características que permiten aseverar lo anterior; una de ellas es que 4 de los 8 lagos más grandes de México son dulces y 4 son salinos, y la otra, que los lagos salinos se encuentran comúnmente en cuencas endorreicas de regiones semiáridas, en los cinturones localizados entre $\operatorname{los} 20^{\circ}$ y $\operatorname{los} 40^{\circ} \mathrm{N}$, las cuales abarcan casi un $65 \%$ del territorio mexicano en las porciones centro y norte.

Los crustáceos son el grupo más diverso de los artrópodos en los cuerpos acuáticos continentales y tienen una distribución amplia, ocupando una extensa variedad de hábitats. A pesar de lo anterior, es grande el desconocimiento acerca de la biodiversidad y ecología de los crustáceos bentónicos, particularmente los que habitan en los lagos salinos mexicanos. Alcocer y Hammer (1998) mencionan el reducido número de taxa (ostrácodos, decápodos, anfípodos y copépodos harpacticoides) que se han citado para el bentos de estos lagos. Sin embargo, también existen algunos registros de notostracos, conchostracos y anostracos, habitantes típicos de lagos playa y/o temporales.

Las recopilaciones recientes sobre algunos grupos de crustáceos epicontinentales, tales como anfípodos e isópodos (Rocha et al., 2008) han hecho notar la falta de registros -por lo menos publicados-, para muchos cuerpos acuáticos del país, incluso para los lagos más grandes de México (p.ej., Chapala y Cuitzeo), y de manera particular en ríos que, desde este punto de vista, son prácticamente desconocidos. Asimismo, se reconoce que en algunos grupos (p.ej., isópodos, Rocha-Ramírez et al., 2009) existe gran endemicidad, lo cual sugiere que al incrementarse el número de cuerpos acuáticos investigados se probaría que la biodiversidad de crustáceos epicontinentales en el país es alta.

La mayoría de los escasos trabajos publicados al respecto son de naturaleza estrictamente taxonómica, por lo cual el objetivo de esta investigación fue reconocer la composición así como la estructura y distribución espacial, tanto litoral como en el gradiente batimétrico, del ensamble de crustáceos bentónicos en Alchichica, un lago salino tropical ubicado en el extremo oriental del altiplano mexicano.

Alchichica (19²4.7' N, 97²4.0' O) es un lago "maar" que se localiza en la cuenca endorreica oriental $\left(4982 \mathrm{~km}^{2}\right)$ en el extremo SE del altiplano mexicano, entre los estados de Puebla, Veracruz y Tlaxcala (Alcocer et al., 2000; Filonov et al., 2006). Esta zona se originó hace aproximadamente un millón de años durante el Cuaternario (Gasca, 1981). El clima de la región es semiárido con una temperatura ambiental media de $12.9^{\circ} \mathrm{C}$, una tasa de evapotranspiración anual de $1690 \mathrm{~mm}$ y una precipitación menor de $500 \mathrm{~mm}$ (Adame et al., 2008; Alcocer et al., 2000).

La forma de la cuenca del lago se aproxima a la de un cilindro; tiene una reducida zona litoral y un talud muy abrupto con depósitos calcáreos denominados "tufa". La tufa se forma cuando el agua subterránea rica en carbonatos emerge en un lago alcalino-sódico, precipitándose los carbonatos en forma de calcita y en menor medida aragonita, conformando un hábitat para especies de hábitos crípticos. Los depósitos de tufa que se presentan en forma de anillo en la periferia del lago semejan, por su aspecto, arrecifes coralinos.

Alchichica es uno de los lagos naturales más profundos de México, su profundidad máxima es de $62 \mathrm{~m}$ y la media de $40.9 \mathrm{~m}$; la forma del lago es circular con un diámetro de $1733 \mathrm{~m}$, un área de $2.3 \mathrm{~km}^{2}$ y un perímetro de $5.1 \mathrm{~km}$ (Filonov et al., 2006). El principal aporte de agua lo recibe a través del manto freático y en menor cantidad por lluvia. Este lago presenta aguas hiposalinas $\left(\approx 8.5 \mathrm{~g} \mathrm{~L}^{-1}\right)$ y un $\mathrm{pH}$ básico $(\approx 9)$, los iones dominantes son sodio y cloruro; son importantes también los bicarbonatos, carbonatos, sulfatos y el magnesio (Vilaclara et al., 1993).

El día de hoy se reconocen las siguientes especies endémicas para Alchichica: el pez aterínido Poblana alchichica (de Buen, 1945), el anfibio ambistomátido Ambystoma taylori (Brandon et al., 1981), el insecto coríxido Krizousacorixa tolteca (Jansson, 1979), el isópodo Caecidotea williamsi (Escobar-Briones y Alcocer, 2002) y la diatomea Cyclotella alchichicana (Oliva et al., 2006).

Dentro de los antecedentes referentes a los crustáceos bentónicos del lago, se encuentran los trabajos de Alcocer et al. (1993, 1998) sobre el bentos litoral; el de Escobar Briones et al. (1998) sobre isótopos estables de carbono; el de Alcocer et al. (2002) sobre la estructura poblacional del anfípodo Hyalella azteca (de Saussure, 1858), así como los de Escobar-Briones y Alcocer (2002) y Alcocer y Escobar-Briones (2007) sobre la descripción y ecología del isópodo endémico Caecidotea williamsi. Por último, Hernández Fonseca (2001) registró la presencia de un género de copépodo harpacticoide (Bryocamptus) como parte del meiobentos litoral de Alchichica.

\section{Materiales y métodos}

El muestro se llevó a cabo en 3 zonas: litoral ( $<3 \mathrm{~m})$, talud (3-30 m) y zona profunda ( $>45 \mathrm{~m})$. En la zona litoral se eligieron 5 estaciones en la periferia del lago, las cuales se ubicaron para cubrir toda la heterogeneidad ambiental 
creada en gran medida por el grado de comunicación entre la zona litoral y la limnética, impuesta por la presencia del anillo de tufa. En el talud, que es donde predominan los depósitos de tufa, se seleccionaron 8 profundidades de muestreo $(3,5,10,13,15,21,25$ y $28 \mathrm{~m})$. Por último, en la extensa zona profunda se establecieron 4 estaciones (con profundidades de 50, 60, 61 y $62 \mathrm{~m}$ ) representando un gradiente de distancia a la costa. La estación más cercana se ubicó a $\approx 175 \mathrm{~m}$ de la orilla y la más lejana, en el centro del lago, a una distancia de $\approx 900 \mathrm{~m}$. La realización de muestreos a profundidades intermedias se complica por la geomorfología del talud y el predominio de fondos duros.

Para los muestreos de la zona litoral y profunda se empleó una draga tipo Ekman $\left(15 \times 15 \mathrm{~cm} ; 0.0225 \mathrm{~m}^{2}\right.$ de área). Para la zona del talud, en la cual la tufa hace imposible utilizar los métodos típicos de muestreo de organismos bentónicos (dragas, nucleadores, redes), el muestreo se realizó mediante técnicas de buceo autónomo (SCUBA), empleando para ello un nucleador manual de 4 $\mathrm{cm}$ de diámetro. Todas las muestras fueron tomadas por triplicado. Es importante aclarar que la ubicación de las estaciones en el perfil estuvo sesgada, debido a que sólo se pudo muestrear en zonas reducidas del cantil donde se presentaron "repisas", en las que se acumula sedimento que permite el crecimiento de vegetación sumergida y/o algas filamentosas bentónicas. La mayor extensión del cantil es sólo el depósito calcáreo a manera de roca con una orientación casi vertical, por lo que ni se acumula sedimento ni se establece vegetación.

Cada muestra se redujo en volumen con lavados in situ a través de una malla de $0.51 \mathrm{~mm}$ para separar la macrofauna (Gray, 1981; Wetzel, 1981). Las muestras se fijaron con alcohol grado 96, adicionado con rosa de Bengala como colorante vital (APHA AWWA y WPFC,1985). En el laboratorio, los organismos se separaron y preclasificaron por grupo; posteriormente, se identificaron en el nivel taxonómico más bajo posible con ayuda de claves generales (Edmonson, 1959; Pennak, 1978) y especializadas (Bronshtein, 1947) para cada grupo. Además, se reconocieron los hábitos alimenticios de cada especie para determinar su posición trófica (gremio trófico) dentro de las comunidades bentónicas.

Finalmente, los organismos de cada especie se cuantificaron para calcular su densidad y riqueza específica.
La biomasa se estimó colocando el material en un horno a $105^{\circ} \mathrm{C}$ hasta alcanzar un peso constante (peso seco), posteriormente se calcinó en una mufla a $550^{\circ} \mathrm{C}$ (pérdida en combustión -LOI-) para obtener el peso seco libre de cenizas y calcular así, la biomasa en unidades de carbono.

\section{Resultados}

De acuerdo con la literatura y el presente estudio, existen 4 especies registradas de crustáceos bentónicos para el lago Alchichica, 1 anfípodo, 1 isópodo y 2 ostrácodos (Cuadro 1). Sin embargo, en este estudio sólo se recolectaron 3 de éstas, estando ausente el isópodo.

Respecto a la ubicación espacial de las diferentes especies dentro del lago (Cuadro 2), Candona sp. fue la única que se encontró en todas las localidades muestreadas y la única presente en la zona profunda. Limnocythere inopinata fue la segunda especie mejor distribuida, presentándose en todas las localidades litorales y en el $62.5 \%$ (5) de las del talud, profundidades que se encuentran justo por encima del límite de la zona anóxica ( $30 \mathrm{~m})$. Hyalella azteca se presentó en 4 de 5 localidades litorales (80\%) y en 3 de 8 del talud (37.5\%).

La densidad de Candona sp. varió desde $115 \pm 106$ org $\mathrm{m}^{-2}$ hasta $35810 \pm 49571 \mathrm{org} \mathrm{m}^{-2}$. Las densidades más elevadas se encontraron en el talud con $14878 \pm 17681$ org $\mathrm{m}^{-2} \mathrm{y}$ las menores se registraron en la zona profunda con $285 \pm 271$ org $\mathrm{m}^{-2}$, seguramente debido a la prolongada presencia de anoxia. La zona litoral presentó densidades intermedias con $8987 \pm 10284$ org $\mathrm{m}^{-2}$.

Limnocythere inopinata varió de ausente a 8356 \pm 12540 org $\mathrm{m}^{-2}$. Las densidades más altas también se encontraron en la zona del talud con $2699 \pm 5666$ org m$^{-2}$, siendo menores en la zona litoral con $1617 \pm 3308$ org $\mathrm{m}^{-2}$. En la zona profunda estuvo ausente.

Las densidades de $H$. azteca variaron de ausente a 10 $809 \pm 10782$ org m$^{-2}$. A diferencia de los 2 taxa anteriores, las mayores densidades se encontraron en la zona litoral con $4065 \pm 7460$ org m$^{-2}$, disminuyendo a $415 \pm 754$ org $\mathrm{m}^{-2}$ en el talud.

En el caso de los ostrácodos, las mayores abundancias se presentaron en la zona del talud. Por el contrario, las mayores densidades de los anfípodos se observaron

Cuadro 1. Listado de crustáceos macrobentónicos registrados para el lago Alchichica, Puebla

\begin{tabular}{llll}
\hline \multicolumn{2}{c}{ Taxon } \\
\hline Amphipoda & Hyalellidae & Hyalella azteca & Saussure (1858) \\
Isopoda & Asellidae & Caecidotea williamsi & Escobar-Briones y Alcocer (2002) \\
Ostracoda & Limnocytheridae & Limnocythere inopinata & Baird (1843) \\
& Candonidae & Candona sp. & Baird (1845) \\
\hline
\end{tabular}


Cuadro 2. Densidad (org $\mathrm{m}^{-2}$ ) de crustáceos macrobentónicos registrados en las zonas litoral (L), talud (T) y profunda (F) del lago Alchichica, Puebla*

\begin{tabular}{|c|c|c|c|c|c|c|c|c|}
\hline Litoral & $L 1$ & $L 2$ & $L 3$ & L4 & L5 & & & \\
\hline & $0.5 \mathrm{~m}$ & $0.5 \mathrm{~m}$ & $0.5 \mathrm{~m}$ & $0.5 \mathrm{~m}$ & $0.5 \mathrm{~m}$ & & & \\
\hline \multirow[t]{2}{*}{ Hyalella azteca } & 1876 & 5815 & 2877 & 0 & 10809 & & & \\
\hline & 3703 & 851 & 5411 & 0 & 10782 & & & \\
\hline \multirow[t]{2}{*}{ Limnocythere inopinata } & 57 & 367 & 3612 & 1876 & 1923 & & & \\
\hline & 205 & 671 & 4148 & 4957 & 2238 & & & \\
\hline \multirow[t]{2}{*}{ Candona sp. } & 4831 & 5020 & 4346 & 9095 & 22812 & & & \\
\hline & 3834 & 4613 & 3084 & 6428 & 16045 & & & \\
\hline \multirow[t]{2}{*}{ Talud } & $T 1$ & $T 2$ & $T 3$ & T4 & $T 5$ & T6 & $T 7$ & $T 8$ \\
\hline & $3 m$ & $5 \mathrm{~m}$ & $10 \mathrm{~m}$ & $13 \mathrm{~m}$ & $15 \mathrm{~m}$ & $21 \mathrm{~m}$ & $25 \mathrm{~m}$ & $28 \mathrm{~m}$ \\
\hline \multirow[t]{2}{*}{ Hyalella azteca } & 0 & 0 & 1326 & 0 & 796 & 0 & 298 & 0 \\
\hline & 0 & 0 & 459 & 0 & 1125 & 0 & 796 & 0 \\
\hline \multirow[t]{2}{*}{ Limnocythere inopinata } & 3183 & 8356 & 2122 & 0 & 2069 & 0 & 0 & 1194 \\
\hline & 1378 & 12540 & 3676 & 0 & 2293 & 0 & 0 & 1688 \\
\hline \multirow[t]{2}{*}{ Candona sp. } & 1008 & 11738 & 5836 & 11141 & 9231 & 9549 & 27255 & 35810 \\
\hline & 5178 & 9711 & 3925 & 0 & 8600 & 0 & 23930 & 49517 \\
\hline \multirow[t]{2}{*}{ Profunda } & $F 1$ & $F 2$ & $F 3$ & F4 & & & & \\
\hline & $50 \mathrm{~m}$ & $60 \mathrm{~m}$ & $61 \mathrm{~m}$ & $62 \mathrm{~m}$ & & & & \\
\hline \multirow[t]{2}{*}{ Candona sp. } & 115 & 148 & 285 & 593 & & & & \\
\hline & 106 & 101 & 157 & 337 & & & & \\
\hline
\end{tabular}

*El primer renglón es el promedio, el segundo renglón es la desviación estándar.

en la zona litoral. Respecto a la biomasa, Candona sp. varió de $0.33 \pm 0.35 \mathrm{mg} \mathrm{C} \mathrm{m}^{-2}$ a $473.8 \pm 647.2 \mathrm{mg} \mathrm{C} \mathrm{m}^{-}$ ${ }^{2}$ (Cuadro 3). Las biomasas más elevadas se encontraron en el talud con $217.29 \pm 235.86 \mathrm{mg} \mathrm{C} \mathrm{m}^{-2}$ y las menores en la zona profunda con $0.94 \pm 0.97 \mathrm{mg} \mathrm{C} \mathrm{m}^{-2}$. La zona litoral presentó biomasas intermedias de $127.57 \pm 177.11$ $\mathrm{mg} \mathrm{C} \mathrm{m}{ }^{-2}$. Respecto a $L$. inopinata, los valores de biomasa variaron de 0 a $21.23 \pm 30.97 \mathrm{mg} \mathrm{C} \mathrm{m}^{-2}$. Los más altos se encontraron en la zona del talud con $7.05 \pm 14.19 \mathrm{mg} \mathrm{C}$ $\mathrm{m}^{-2} \mathrm{y}$ en la litoral con $5.09 \pm 12.09 \mathrm{mg} \mathrm{C} \mathrm{m}^{-2}$. En la zona profunda estuvo ausente.

Por último, las biomasas de $H$. azteca variaron entre 0 y $402.80 \pm 1328.94 \mathrm{mg} \mathrm{C} \mathrm{m}^{-2}$. Las mayores biomasas se encontraron en la zona litoral con $85.37 \pm 100.02 \mathrm{mg} \mathrm{C}$ $\mathrm{m}^{-2}$, disminuyendo en el talud con $3.38 \pm 6.75 \mathrm{mg} \mathrm{C} \mathrm{m}^{-2}$. La biomasa se relaciona con la talla de los organismos, su abundancia y la presencia o ausencia de esqueletos más calcificados.

\section{Discusión}

Es probable que de las 3 especies encontradas, el anfípodo sea una especie nueva de Hyalella aún sin describir, ya que, como González y Watling (2002) mencionan, $H$. azteca es un complejo de especies distribuido en Norteamérica, Centroamérica y norte de Sudamérica. Respecto a los ostrácodos, de acuerdo con Palacios-Fest (com. pers.), la Candona registrada en Alchichica no corresponde a ninguna de las especies descritas para el género, por lo que seguramente también corresponde a una especie nueva aún por describir. El isópodo es una especie endémica de Alchichica (EscobarBriones y Alcocer, 2002) y el primer asélido conocido que habita aguas salinas epicontinentales en el continente americano (Alcocer y Escobar-Briones, 2007). Resulta interesante que a pesar de su limitada riqueza taxonómica, Alchichica sea un sitio de numerosos endemismos con 5 especies endémicas registradas hasta el momento y 2 más en potencia.

Es importante mencionar que no es extraño que $C$. williamsi no haya sido recolectada; este organismo, como Alcocer y Escobar-Briones (2007) informan, es de hábitos crípticos y vive preferentemente entre las hendiduras de la tufa y no así en el sustrato lodoso que caracteriza las estaciones muestreadas. Se puede concluir que el tipo 
Cuadro 3. Biomasa $\left(\mathrm{mg} \mathrm{C} \mathrm{m}^{-2}\right)$ de crustáceos macrobentónicos registrados en las zonas litoral $(\mathrm{L})$, talud $(\mathrm{T})$ y profunda $(\mathrm{F})$ del lago Alchichica, Puebla*

\begin{tabular}{|c|c|c|c|c|c|c|c|c|}
\hline Litoral & $L 1$ & $L 2$ & $L 3$ & L4 & L5 & & & \\
\hline & $0.5 \mathrm{~m}$ & $0.5 \mathrm{~m}$ & $0.5 \mathrm{~m}$ & $0.5 \mathrm{~m}$ & $0.5 \mathrm{~m}$ & & & \\
\hline \multirow[t]{2}{*}{ Hyalella azteca } & 4.23 & 402.80 & 4.47 & 0 & 30.13 & & & \\
\hline & 7.75 & 1328.94 & 6.94 & 0 & 34.37 & & & \\
\hline \multirow[t]{2}{*}{ Limnocythere inopinata } & 0.12 & 0.94 & 9.83 & 5.4 & 9.49 & & & \\
\hline & 0.43 & 1.73 & 11.6 & 14.51 & 17.84 & & & \\
\hline \multirow[t]{2}{*}{ Candona sp. } & 75.38 & 69.41 & 42.9 & 114 & 366.2 & & & \\
\hline & 85.95 & 70.55 & 37.8 & 83.49 & 281 & & & \\
\hline \multirow[t]{2}{*}{ Talud } & $T 1$ & $T 2$ & $T 3$ & $T 4$ & $T 5$ & T6 & $T 7$ & $T 8$ \\
\hline & $3 m$ & $5 \mathrm{~m}$ & $10 \mathrm{~m}$ & $13 \mathrm{~m}$ & $15 \mathrm{~m}$ & $21 \mathrm{~m}$ & $25 \mathrm{~m}$ & $28 m$ \\
\hline \multirow[t]{2}{*}{ Hyalella azteca } & 0 & 0 & 10.8 & 0 & 5.93 & 0 & 3.38 & 0 \\
\hline & 0 & 0 & 4.75 & 0 & 8.18 & 0 & 6.75 & 0 \\
\hline \multirow[t]{2}{*}{ Limnocythere inopinata } & 8.71 & 21.23 & 5.62 & 0 & 5.57 & 0 & 0 & 6.41 \\
\hline & 3.77 & 30.97 & 7.74 & 0 & 6.33 & 0 & 0 & 0 \\
\hline \multirow[t]{2}{*}{ Candona sp. } & 147.9 & 159.1 & 95.2 & 215.9 & 151.5 & 129 & 395.7 & 473.8 \\
\hline & 70.73 & 123.68 & 43.7 & 0 & 119.4 & & 330.3 & 647.2 \\
\hline \multirow[t]{2}{*}{ Profunda } & $F 1$ & $F 2$ & $F 3$ & $F 4$ & & & & \\
\hline & $50 \mathrm{~m}$ & $60 \mathrm{~m}$ & $61 \mathrm{~m}$ & $62 \mathrm{~m}$ & & & & \\
\hline \multirow[t]{2}{*}{ Candona sp. } & 0.33 & 0.44 & 0.92 & 2.07 & & & & \\
\hline & 0.35 & 0.35 & 0.56 & 1.19 & & & & \\
\hline
\end{tabular}

*El primer renglón es el promedio, el segundo renglón es la desviación estándar.

de muestreo empleado no es el adecuado para reconocer la presencia de la especie. Más aún, los mismos autores mencionan que este isópodo se distribuye desde la superficie hasta los $30 \mathrm{~m}$ de profundidad, límite impuesto por la presencia de oxígeno disuelto.

La variación de las densidades obtenidas en $H$. azteca $\left(\geq 10,000\right.$ org $\left.\mathrm{m}^{-2}\right)$ ya había sido registrada previamente por Alcocer et al. (2002); tanto Pennak (1978) como Covich y Thorp (1991) mencionan que los anfípodos pueden ser "increíblemente abundantes"; alcanzan densidades de varios miles de organismos por metro cuadrado, particularmente en sustratos complejos donde la comida detrítica es abundante y están menos expuestos a la depredación.

En el caso de los ostrácodos, las mayores abundancias se presentaron en la zona del talud, lo cual podría ser debido a que ésta constituye una zona de transición entre hábitats, donde el impacto de parámetros ambientales no tiene un efecto negativo en la presencia de organismos, especialmente para Limnocythere (Carbonel et al., 1990).

La amplia variación en las densidades registradas en el talud, al igual que en otros estudios de ostrácodos (Griffiths et al., 1993), sugiere que los organismos tienen una distribución contagiosa; sin embargo, la densidad promedio disminuye marcadamente conforme se incrementa la profundidad, tal y como se informa para otros sistemas lacustres (p.ej., Holopainen y Paasivirta, 1977). Por el contrario, las mayores densidades de los anfípodos se observaron en la zona litoral; la preferencia por aguas someras es una característica común en este grupo de organismos (Pennak, 1978).

La distribución de taxa en el lago muestra que existe una diferencia estructural de hábitats; la zona litoral presenta mayor heterogeneidad de hábitats y más recursos alimenticios; por otro lado, la zona profunda representa un hábitat más homogéneo, en donde el oxígeno disuelto se agota por un largo periodo de tiempo. Seguramente por lo anterior, los crustáceos bentónicos están representados por un sólo taxon que se ha adaptado a las condiciones adversas. Este patrón de distribución es típico del bentos de agua dulce, donde las densidades máximas se presentan en la zona litoral y disminuyen al aumentar la profundidad (Diggins y Thorp, 1985).

El género Candona es abundante en los sedimentos de una amplia variedad de hábitats acuáticos; sus especies se encuentran en la zona litoral con abundancias elevadas 
que disminuyen conforme se profundiza, hasta los $300 \mathrm{~m}$ de profundidad (Griffiths y Martin, 1993). Candona se considera un género común en hábitats cuya temperatura no excede los $18^{\circ} \mathrm{C}$ (Hartmann y Hiller, 1977) y tiene una gran capacidad para sobrevivir largos periodos en los fondos anóxicos de lagos estratificados (Griffiths, 1995; Pennak 1978), lo cual explica claramente su presencia en la zona profunda de Alchichica.

El género Limnocythere tiene una distribución amplia en la zona litoral y disminuye a profundidades mayores (Niinemets, 1999), donde la falta de oxígeno disuelto parece ser el factor limitante para la mayor parte de los crustáceos, tal y como se encontró en Alchichica. La distribución de este género se restringe a hábitats oligotróficos (Scharf, 1981) y es tolerante a aumentos de salinidad (Savolainen y Valtonen, 1984; Frenzel, 1991). Suele ser un género muy sensible a cambios en el grado trófico de un sistema (Griffiths, 1995). En particular, L. inopinata es una especie relativamente común en cuerpos acuáticos epicontinentales e indicador de aguas con elevada alcalinidad (Löffler, 1991) y tolerante a la salinidad (Listera et al., 1991).

Adicionalmente, la presencia de ambos géneros de ostrácodos, Candona y Limnocythere, tiene una alta correlación con la concentración disponible de magnesio y calcio en el sistema (Bunbury y Gajewski, 2005); ambos cationes son abundantes en Alchichica (Armienta et al., 2008), particularmente el magnesio con $431 \mathrm{mg} \mathrm{L}^{-1}$.

Con frecuencia, los miembros más importantes de las comunidades litorales de los cuerpos de agua epicontinentales son los anfípodos (Pennak, 1978). Por su tamaño buscan sitios que ofrezcan protección contra sus depredadores, habitando principalmente entre las macrofitas u otro tipo de vegetación acuática sumergida (Covich y Thorp, 1991). Asimismo, se les encuentra en la capa más superficial de sedimentos suaves de cuerpos acuáticos de aguas frías y no contaminadas donde pueden consumir microfitobentos y debris de origen vegetal. Hyalella azteca es un típico organismo de aguas dulces que puede tolerar condiciones de salinidad entre 5.5 y 22.5 $\mathrm{g} \mathrm{L}^{-1}$ (Timms et al., 1986).

Respecto al análisis trófico, el ensamble de crustáceos bentónicos del lago Alchichica se compone de especies herbívoras ( $H$. azteca), omnívoras ( $C$. williamsi) y bacterivoras (L. inopinata y Candona sp.), con un claro predominio de los herbívoros hacia la zona somera y bien iluminada, donde se presenta la vegetación tanto micro (p.ej., algas filamentosas bentónicas) como macrofítica (Ruppia maritima) y de los bacterívoros hacia las más profundas y oscuras, donde se deposita y descompone el material orgánico exportado desde la zona pelágica.

\section{Agradecimientos}

A Laura Peralta, Luis A. Oseguera, Armando Rodríguez y a Virginia E. Urbieta y su equipo de buceo SCUBA, por su apoyo en el campo y laboratorio.

\section{Literatura citada}

Adame, M. F., J. Alcocer y E. Escobar. 2008. Size-fractionated phytoplankton biomass and its implications for the dynamics of an oligotrophic tropical lake. Freshwater Biology 53:2231.

Alcocer, J. y W. D. Williams. 1993. Lagos salinos mexicanos. In Biodiversidad marina y costera de México, S. I. SalazarVallejo y N. E. González (eds.). Comisión Nacional para el Conocimiento y Uso de la Biodiversidad / y Centro de Investigaciones de Quintana Roo (CIQRO), Chetumal. p. 849-865.

Alcocer, J., A. Lugo, S. Estrada, M. Ubeda y E. Escobar. 1993. La macrofauna bentónica de los axalapazcos mexicanos. Actas del VI Congreso Español de Limnología 33:409-415.

Alcocer, J. y U. T. Hammer. 1998. Saline lake ecosystems of Mexico. Aquatic Ecosystem Health and Management 1:291315.

Alcocer, J., E. Escobar, A. Lugo y L. Peralta. 1998. Littoral benthos of the saline crater-lakes of the basin of Oriental, Mexico. International Journal of Salt Lake Research 7:87108.

Alcocer J., A. Lugo, E. Escobar, M. R. Sánchez y G. Vilaclara. 2000. Water column stratification and its implications in the tropical warm monomictic Lake Alchichica, Puebla, Mexico. Verhandlungen Internationale Vereinigun für theoretische und angewandte Limnologie 27:3166-3169.

Alcocer, J., E. Escobar, L. Peralta y F. Álvarez. 2002. Population structure of the macrobenthic amphipod Hyalella azteca Saussure (Crustacea: Peracarida) on the littoral zone of six crater lakes. In Modern approaches to the study of Crustacea, E. Escobar-Briones y F. Álvarez (eds.). Kluwer, Nueva York. p. 111-115.

Alcocer, J. y E. Escobar-Briones. 2007. On the ecology of Caecidotea williamsi Escobar-Briones y Alcocer (Crustacea: Isopoda: Asellidae) from Alchichica saline lake, Central Mexico. Hydrobiologia 576:103-109.

APHA, AWWA y WPCF, 1985. Standard methods for the examination of water and wastewater. American Public Health Association, New York. 1193 p.

Armienta, M. A., G. Vilaclara, S. de la Cruz-Reyna, S. Ramos, N. Ceniceros, O. Cruz, A. Aguayo y F. Arcega-Cabrera. 2008. Water chemistry of lakes related to active and inactive Mexican volcanoes. Journal of Volcanology and Geothermal Research 178:249-258.

Brandon, R. A., E. J. Maruska y W. T. Rumph. 1981. A new species of neotenic Ambystoma (Amphibia, Caudata) endemic to Laguna Alchichica, Puebla; México. Bulletin of the Southern California Academy of Sciences 80:112-125.

Bronshtein, Z. S. 1947. Ostracoda presny vod. In Fauna S.S.S.R., 
Rakoobraznye II. 1. Zool. Instituta Akademii Nauk SSSR, n.s. 31:339

Bunbury, J. y K. Gajewski. 2005. Quantitative análisis of freshwater ostracode assemblages in Southwestern Yukon Territory, Canada. Hidrobiologia 545:117-128.

Carbonel, P., P. Mourguiart y J. P. Peypouquet. 1990. The external mechanisms responsible for morphological variability in recent Ostracoda: seasonality and biotype situation: an example from Lake Titicaca. In Ostracoda and globlal events, R. Whatley y C. Maybury (eds.). British Micropaleontological Society Publication Series. Cambridge. University Press. p. 331-340.

Covich, A. P. y J. H. Thorp. 1991. Crustacea: Introduction and Peracarida. In Ecology and classification of North American freshwater invertebrates, J. H. Thorp y A. P. Covich (eds.). Academic, San Diego, California. p. 665-690.

de Buen, F. 1945. Investigaciones sobre ictiología mexicana. I. Atherinidae de aguas continentales de México. Anales del Instituto de Biología, Universidad Nacional Autónoma de México 16:475-532.

de Saussure, H. 1858. Mémoire sur divers crustacés nouveaux des Antilles et du Mexique. Mémoires de la Société de physique et d'histoire naturelle de Genève 14:417-496.

Diggins, M. R. y J. H. Thorp. 1985. Winter-spring depth distribution of Chironomidae in a southeastern reservoir. Freshwater Invertebrate Biology 4:8-21.

Edmonson, W. T. 1959. Fresh-water biology. Wiley, New York. $1248 \mathrm{p}$.

Escobar-Briones, E. y J. Alcocer. 2002. Caecidotea williamsi (Crustacea: Isopoda: Asellidae), a new species from a saline crater-lake in the eastern Mexican Plateau. Hydrobiologia 477:93-105.

Escobar Briones, E., J. Alcocer, E. Cienfuegos y P. Morales. 1998. Carbon stable isotopes of pelagic and littoral communities in Alchichica crater-lake, Mexico. International Journal of Salt Lake Research 7:345-355.

Frenzel, P. 1991. Die Ostracodenfauna der tieferen Teile der Ostsee-Boddengewässer Vorpommerns. Meyniana 43:151175.

Filonov, A., I. Tereshchenko y J. Alcocer. 2006. Dynamic response to mountain breeze circulation in Alchichica, a crater lake in Mexico. Geophysical Research Letters 33. L07404, DOI: 10.1029/2006GL025901

Gasca, D. A. 1981. Algunas notas de la génesis de los lagoscráter de la cuenca de Oriental. Puebla-Tlaxcala-Veracruz, Colección Científica Prehistoria 98. Instituto Nacional de Antropología e Historia, México, D.F. 55 p.

González, E. R. y L. Watling. 2002. Redescription of Hyalella azteca from its type locality, Vera Cruz, Mexico (Amphipoda: Hyalellidae). Journal of Crustacean Biology 22:173-183.

Gray, J. S. 1981. The ecology of marine sediments. Cambridge Studies in Modern Biology 2. Cambridge University Press. $185 \mathrm{p}$.

Griffiths, H. I., D. S. Martin, A. J. Shine y J. G. Evans. 1993. The ostracod fauna (Crustacea, Ostracoda) of the profundal benthos of Loch Ness. Hydrobiologia 254:111-117.

Griffiths, H. I. 1995. The application of freshwater Ostracoda to the reconstruction of Late Quaternary Palaeoenvironments in North-western Europe. PhD Thesis, University of Wales, Cardiff., 449 p.

Griffiths, H. I. y D. S. Martin. 1993. The spatial distribution of benthic ostracods in the profundal zone of Loch Ness. Collected papers on Loch Ness research initiated by the Loch Ness and Morar Project. Scottish Naturalist 105:137-147.

Hartmann, G. y F. Hiller. 1977. Beitrag zur Kenntnis der Ostracodenfauna des Harzes und seines nördlichen Vorlandes (unter besonderer Berücksichtigung des Männchens von Candona candida). Naturwissenschaftlicher Verein Goslar 125:99-116.

Hernández Fonseca, M. del C. 2001. Densidad y biomasa de la meiofauna de la zona litoral de los lagos-cráter Alchichica, Quechulac y Tecuitlapa, Puebla, México. Tesis, Facultad de Estudios Superiores Iztacala, Universidad Nacional Autónoma de México, México, D.F. 51 p.

Holopainen, I. J. y L. Paasivirta. 1977. Abundance and biomass of the meiozoobenthos in the oligotrophic and mesohumic Lake Pääjärvi. Annales Zoologici Fennici 14:124-134.

Jansson, A. 1979. A new species of Krizousacorixa from Mexico (Heteroptera, Corixidae). Pan-Pacific Entomologist 55:258260.

Listera G. S., K. Keltsb, Chen Ke Zaoc, Jun-Qing Yua y F. Niessena. 1991. Lake Qinghai, China: closed-basin like levels and the oxygen isotope record for ostracoda since the latest Pleistocene. Palaeogeography, Palaeoclimatology, Palaeoecology 84:141-162.

Löffler, H. 1991. Paleolimnology of Neusiedlersee, Austria. Hydrobiologia 214:229-238.

Niinemets, E. 1999. Ostracode paleoecology and stable isotopes from Lake Peipsi, Estonia. MSc. Thesis, Institute of Geology, University of Tartu [Estonia]. $43 \mathrm{p}$.

Oliva, M. G., A. Lugo, J. Alcocer y E. A. Cantoral-Uriza. 2006. Cyclotella alchichicana sp. nov. from a saline Mexican lake. Diatom Research 21:81-89.

Pennak, R. W. 1978. Fresh-water invertebrates of the United States. Wiley, New York. 803 p.

Rocha, A., L. Peralta y J. Alcocer. 2008. Cap. 4. Anfípodos e isópodos de aguas epicontinentales de México. In Crustáceos de México: estado actual de su conocimiento, F. Álvarez y G. Rodríguez Almaraz (eds.). Universidad Autónoma de Nuevo León, Monterrey. p. 53-79.

Rocha-Ramírez, A., F. Álvarez, J. Alcocer, R. Chávez-López y E. Escobar-Briones. 2009. Lista anotada de los isópodos acuáticos epicontinentales de México (Crustacea: Isopoda). Revista Mexicana de Biodiversidad 80:615-631.

Savolainen, I. y T. Valtonen. 1984. Ostracods of the north-eastern Bothnian Bay and population dynamics of the principal species. Aquilo, Seria Zoologica 22(1983):69-76.

Scharf, B. W. 1981. Zur rezenten Muschelkrebsfauna der Eifelmaare (Crustacea: Ostracoda). Mitteilungen der Pollichia 68:185-204.

Shiklomanov, I. A., 1990. Global water resources. Natural Resources 26:34-43.

Timms, B. V., U. T. Hammer y J. W. Sheard. 1986. A study of benthic communities in some saline lakes in Saskatchewan and Alberta, Canada. Internationale Revue der gesatmen Hydrobiologie 71:759-777. 
Vilaclara, G., M. Chávez, A. Lugo, H. González y M. Gaytán. 1993. Comparative description of crater-lakes basic chemistry in Puebla State, Mexico. Verhandlungen Internationale
Vereinigun für theoretische und angewandte Limnologie 25:435-440.

Wetzel, R. G. 1981. Limnología. Omega, Barcelona. 680 p. 